\title{
A Computer-Assisted Method for the Study of the Trabecular Bone of the Distal Radius on Conventional Radiographs
}

\author{
Donatella Trippi, ${ }^{*}$ Massimo Chimenti, $\uparrow$ and Renzo Bozzi†
}

\begin{abstract}
The procedure described is based on the acquisition and processing of $x$-rays of the distal radius obtained under standard conditions. An x-ray was obtained of the forearm together with an aluminum step wedge to automatically normalize the photometric values of the bone with respect to the photometric values of the reference aluminum wedge. Densitometric values for thickness (T) and a coarseness parameter (C) that depends on the trabecular bone pattern are measured on interactively selected rows and regions of interest (ROIs) of the digital image. Twenty-five women were examined and two different measurements were performed. The first measurement considers $C$ in three sites of the radial epiphisis. The trabecular bone coarseness appears to increase from the distal to the veryvery distal site and the value of $\mathrm{C}$ in the very distal site, which is located $1 \mathrm{~cm}$ distally to the distal one tenth of the radius, seems to be related to the pathological variations more than the value of $\mathrm{C}$ in the other sites. The second measurement is the $\mathrm{C} / \mathrm{T}$ ratio of eight $\mathrm{RO}$ is of 15 patients: five healthy and 10 osteoporotic women. This ratio is significantly different for the two groups in all the eight ROls and the variations are particularly significant at 6 to $12 \mathrm{~mm}$ from the subchondral line. Copyright 1993 by W.B. Saunders Company
\end{abstract}

KEY WORDS: digital image processing, computerassisted radiology, bone density, osteoporosis, trabecular bone pattern.

$\mathbf{T}$ HE METHODS MOST commonly used to diagnose osteoporosis are based on measurements of the content and density of the bone mineral. Therefore, such methods refer only to the type of material composing the skeleton. However, another important parameter that can be used to determine the strength of the skeleton is represented by the geometry or architecture of the bone structure.

Methods previously used in radiography, such as those described in Cann, ${ }^{1}$ Griffiths and Virta$\mathrm{ma}^{2}$ and, in particular, methods that involved studies of the trabecular structure must now be

From the Cattedra di Radiologia, Università di Pisa, *and the Istituto di Elaborazione della Informazione, CNR, Pisa, $\dagger$ Italy.

Address reprint requests to Donatella Trippi, $M D$, Istituto di Radiologia, Università di Pisa, Via Roma, 57, 56100 Pisa, Italy.

Copyright (c) 1993 by W.B. Saunders Company

0897-1889/93/0602-0003\$03.00/0 considered obsolete. However, recently, the use of noninvasive procedures to process standard $\mathrm{x}$-rays has been proposed, even though such procedures have not yet been applied clinically in the quantification of the geometric characteristics of bone structures. For example, systems for the computerized analysis of bone structures are now applied in histomorphometry. ${ }^{3,4}$

Noninvasive measuring procedures using a number of different image processing techniques have been tried, such as pattern recognition, Fourier transform, and run-length analysis. Rockoff et $\mathrm{al}^{5}$ used a pattern recognition method to measure the mean trabecular signal strength and the angular distribution of the trabeculae, showing an alteration in the mainly longitudinal structure of the trabeculae of the radius and a total loss of signal for osteoporotic patients. On the other hand, the run-length method, applied on high definition computed tomography (CT) images, has made it clear that a precise simulation of the structure is extremely difficult given the complexity of the data to be analyzed and the fact that these data are to some extent negatively influenced by the partial volume effect. ${ }^{6}$

The pattern recognition methods mentioned above are generally used, at least in theory, to derive the parameters needed for a total description of the type of trabecular texture being examined. However, the method proposed in this article only measures the degree of coarseness, without determining the spatial orientation of the trabeculae. For this reason, the method has been applied on a bone portion whose tissue presents a reduced degree of anisotropy, ie, with the trabeculae in a mainly longitudinal direction.

A similar measuring method was used by Trouerbach et al to evaluate the bone structure of the phalanges by analyzing some rows of a conventional $\mathrm{x}$-ray, digitized by means of a linear microdensitometer. ${ }^{7}$

This article describes a procedure that has been developed for the digital processing on conventional $x$-rays of the distal portion of the forearm. The aspect of the trabecular bone in 


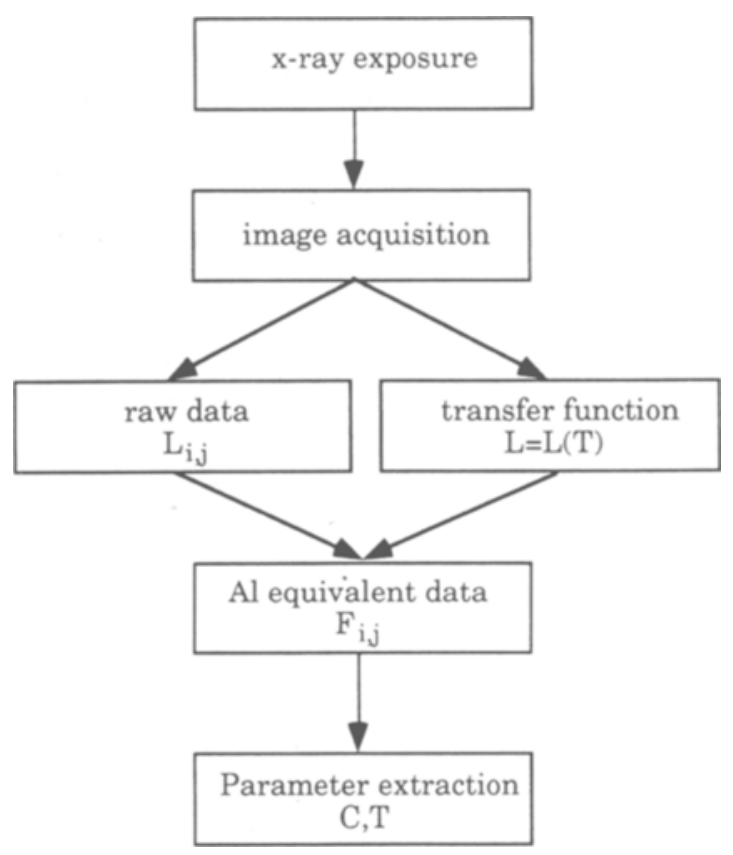

Fig 1. Overall schema of the procedure for trabecular bone pattern analysis.

this site gives a more accurate indication of the calcium balance than the cortical bone of the diaphysis $^{\gamma}$ and also permits a more accurate estimate of the bone mass of the spine. ${ }^{9}$

We have developed an experimental procedure for the in vivo determination of the balance of the trabecular bone in the bone mass of the distal epiphysis of the radius. The procedure is used for data gathering and, after adequate clinical testing, could be applied, together with densitometry techniques and laboratory investi- gations, to the diagnosis of diseases that involve the loss of bone mineral content.

\section{MATERIALS AND METHODS}

A schema of the procedure that we have developed is given in Fig 1.

The distal portion of the forearm is exposed to the $\mathrm{x}$-ray using a high definition film-screen system with a standard technique $(25 \mathrm{~mA}$ and $40 \mathrm{kV}$ at $70 \mathrm{~cm}$ ); an aluminum wedge with 21 .5 - $\mathrm{mm}$ steps is also included in each image.

The $\mathrm{x}$-rays have been digitized using an image scanner that measures the optical transparence. The scanner (Fig 2) includes an optoelectronic transducer consisting of an array of 2,048 photodiodes. The transducer covers a field of $25.6 \mathrm{~cm}$ with a resolution of $125 \mu \mathrm{m}$. The $\mathrm{x}$-ray film is translated by a mechanism that is activated by a step motor to obtain a resolution of $125 \mu \mathrm{m}$ in the mechanical scanning direction as well. The $\mathrm{x}$-ray is illuminated by a linear light source consisting of a fluorescent tube that is aligned and fixed with respect to the optoelectronic transducer. In this way, each photodiode receives the light transmitted by the $x$-ray. An analog to digital converter connected to the output of the control circuitry of the array of photodiodes converts the video signal into an 8-bit digital value. Thus, for each pixel on the $x$-ray, we have a raw value $L_{i, j}$ where $L=0,1,2$, .. 255 is the luminance of the pixel, proportional to the transparency of the $\mathrm{x}$-ray, and $\mathrm{i}, \mathrm{j}=$ $0,1,2, \ldots 2047$ are the scanning coordinates.

Following a commonly adopted method, ${ }^{10}$ the
Fig 2. The image processing workstation consisting of a PC with pictorial and alphanumerical displays (left side) and of the xy scanner for radiograms acquisition (right side).

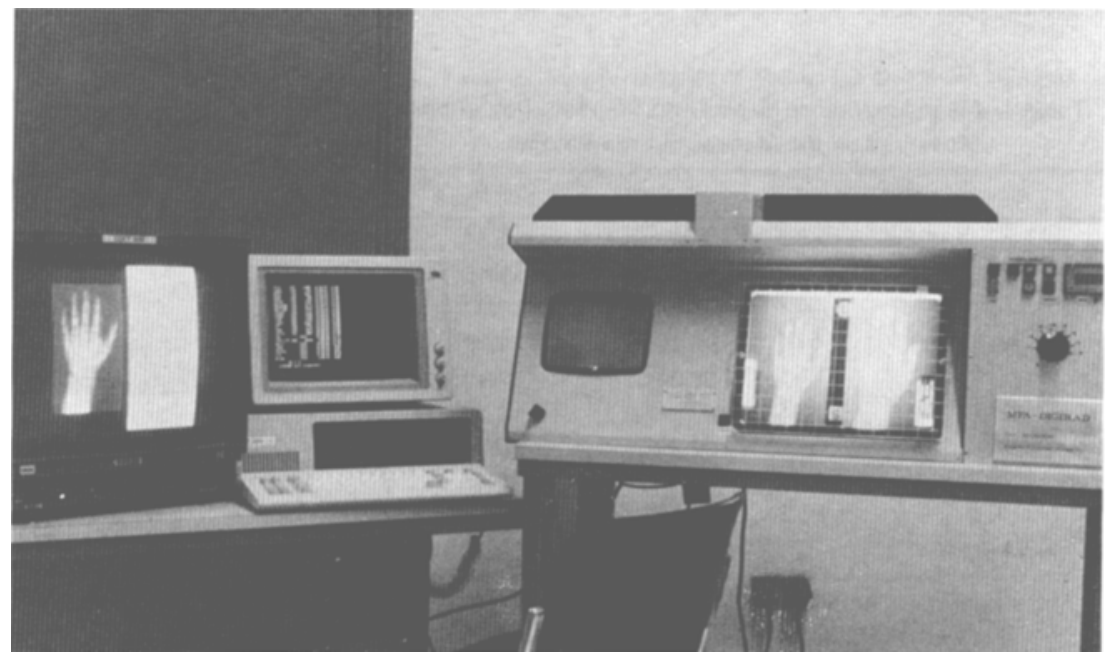


raw data $L_{i, j}$ are converted into normalized data $F_{i, j}$, using the calibration curve $L=L(T)$ obtained by the acquisition data of the aluminum wedge. The curve $L(T)$ is constructed by interpolation, measuring the average transparency of the $\mathrm{x}$-ray over areas of $16 \times 16$ pixel in correspondence with each step of the wedge. Given $T_{j}$, where $j=1,2, \ldots 20$, the thickness of the wedge in correspondence with the $j$-th step, the average value $L_{j}$ can be derived. The value $L_{i, j}$, obtained on the bone image, is converted into an equivalent thickness of aluminium using the inverse function $T=T(L)$. Because the transfer function is determined separately for each $\mathrm{x}$ ray, any artifacts (caused by variations in the exposure conditions or in the exposure-density curve of the film used) are eliminated and normalized data are obtained.

In the above operation, no allowance is made for the radiographic contribution of the soft tissues. However, a partial compensation is made in the processing procedure. The average luminance values, derived on the steps of the reference wedge, are provided in Table 1 . The table also reports the standard deviation (SD) of the measuring values on each range of interest. The SD can be taken as an estimate of the accuracy of the value measured for the equivalent thickness (with probable error of 0.7 SD). ${ }^{11}$

The ROIs in the digital image generated by matrix $F_{i, j}$ are selected interactively; in this case these regions are located in the distal portion of the radius. The measuring sites are also selected interactively. An example is shown in Fig 3. Figure 4 shows the graph $F_{i}$ given by the values

Table 1. Mean Luminance Values and Standard Deviations Measured on the Aluminum Step Wedge

\begin{tabular}{ccc}
\hline Aluminum Step & $\mathrm{L}$ & $\mathrm{SD}$ \\
\hline 13 & 114.0 & 1.34 \\
12 & 105.5 & 1.45 \\
11 & 93.6 & 1.37 \\
10 & 81.1 & 1.21 \\
9 & 66.0 & 1.14 \\
8 & 49.0 & 1.46 \\
7 & 32.3 & 0.79 \\
6 & 18.7 & 0.46 \\
5 & 8.5 & 0.58 \\
4 & 2.06 & 0.23 \\
\hline
\end{tabular}

Abbreviations: L, luminance; SD, standard deviation.

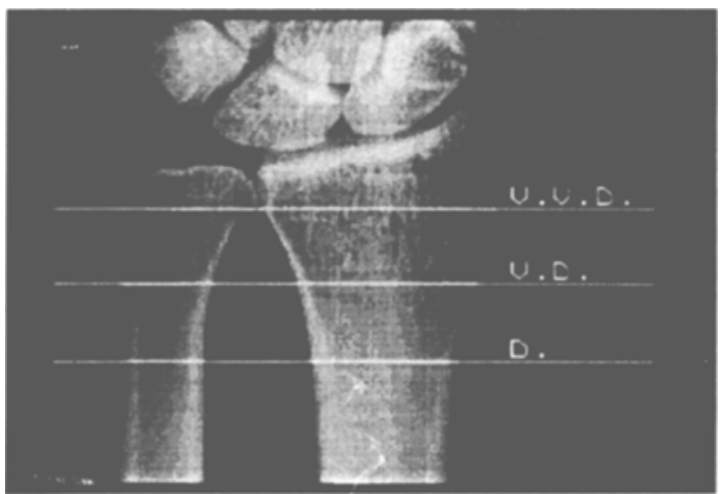

Fig 3. Selection of measuring sites: WD, very-very distal line: VD very distal line; D distal line.

of a row of the image resulting from a scan orthogonal to the axis of the radius. The $x$ axis of the graph gives the scan coordinates from 0 to 511 gray levels, in the ordinate values represent the equivalent thickness of the aluminum wedge in relative units from 0 to 255 gray levels.

Examining the portion of the graph corresponding to the radius, we find the first section to have a moderate gradient in correspondence with the soft tissues, whereas the next section has a steeper gradient only in correspondence with the bone cdge and a positive peak in correspondence with the projection of the cortical structure. The graph also shows a section that is included between the two peaks of the cortex, with oscillations of varying strengths

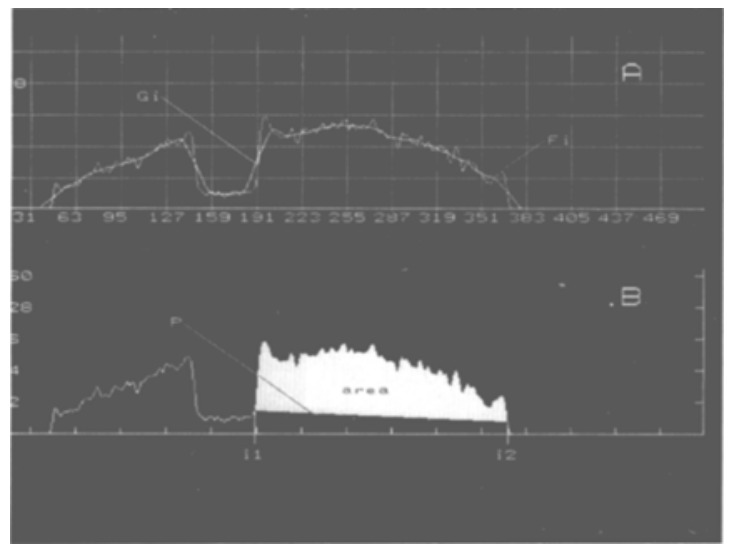

Fig 4. (A) Graph of a row of the acquired image (curve $F_{i}$ ) and the filtered signal (curve $G_{i}$ ), used to calculate parameter $C$ (see text). (B) Graph of the acquisition signal $F_{i}$ and the pedestal $P_{i}$ identified by the scan coordinates $i_{1}$ and $i_{2}$ the area in white between the two signals is used to calculate parame. ter T. 
and, finally, a trend that is symmetrical to the initial section.

The coarseness (C) and thickness ( $\mathrm{T}$ ) parameters are calculated in the following way: The input signal $F_{i}$ is convolved with a box function, $H$, with length $n=19$, and a smoothed signal $\mathrm{G}_{\mathrm{i}}=\mathrm{F} * \mathrm{H}$ is obtained. $\mathrm{C}$ is the root mean square value of the signal given by the difference between $F_{i}$ and $G_{i}$ :

$$
C=\sqrt{\frac{1}{i_{2}-i_{1}+1} \sum_{i_{1}}^{i_{2}}\left(F_{i}-G_{i}\right)^{2}}
$$

where $i_{l}$ and $i_{2}$ are the scanning coordinates in correspondence with the edges of the radius.

The operation indicated by equation 1 is equivalent to the measurement of the power spectrum of $F_{i}$ in a frequency interval determined by the filter function: $\mathrm{C}$ is thus dependent on the high frequency components of $F_{i}$ which mainly result from the trabecular structure of the bone. ${ }^{7}$

To measure $T$, the acquisition data $F_{i, j}$ must be transformed into a signal that is only dependent on the attenuation produced by the skeletal structure.

Consider a graph $F_{i}$; for $i=i_{1}$ and $i=i_{2}, F_{i}$ is only dependent on the attenuation of the soft tissues (skin, muscles, etc) whereas for $i_{1}<i<$ $i_{2}, F_{i}$ depends on the attenuation due to both the bone and the soft tissues surrounding it. Assuming the thickness of the soft tissues to be constant or to vary linearly, the content of these tissues can be eliminated by a subtraction. ${ }^{12}$

We subtract from $F_{i}$ a pedestal of height $P_{i}$ (the white area in Fig 4B). This pedestal is given by the straight line segment between points $F_{i_{1}}$ and $F_{i_{2}}$ :

$$
\begin{aligned}
& P_{i}=i \frac{F_{i_{2}}-F_{i_{1}}}{i_{2}-i_{1}}+\frac{i_{2} F_{i_{1}}-i_{1} F_{i_{2}}}{i_{2}-i_{1}} \\
& \text { for } i_{1} \leq i<i_{2}
\end{aligned}
$$

$\mathrm{T}$ is the mean value of the signal obtained by subtraction and is defined by:

$$
\mathrm{T}=\frac{1}{i_{2}-i_{1}+1} \sum_{i_{1}}^{i_{2}}\left(F_{i}-P_{i}\right)
$$

To evaluate the reliability of our hypothesis, an x-ray of a forearm dried bone and an x-ray of the same bone laid over a $3-\mathrm{mm}$ thick aluminium plate were both analyzed. The equivalent thickness was derived using equation 3 and was found to be $2.95 \mathrm{Almm}$ for the dried bone and $3.21 \mathrm{Almm}$ for the dried bone placed over the aluminium plate. Table 2 reports the results obtained measuring known thicknesses of aluminium. The table presents the values of $\mathrm{T}$ derived from equation 3 , the values of bone mineral density (BMD) supplied by the dual photon absorptiometry (DPA) and the values for $\mathrm{k}=$ $\mathrm{BMD} / \mathrm{T}$. The mean values of $\mathrm{T}$ are in good agreement with the actual thicknesses of the aluminum. Furthermore, the values derived from the image analysis and those resulting from the densitometry are comparable. The mean value for $k$ is 141 , with an SD of 1.19.

Under the above assumptions, $\mathrm{T}$ is mainly determined by the attenuation produced by the skeletal tissue. The value of $\mathrm{T}$ depends on the product of the linear attenuation coefficient $\mu$ of the crossed tissue for the corresponding thickness; because $\mu$ is proportional to the tissue density, $T$ is a measure of the product of the density and the thickness of the radiographed tissue. ${ }^{12}$ Equal values of $T$ can be obtained by exposing bone tissues of high density and low thickness to x-rays, or by exposing bone tissues of low density and high thickness to $\mathrm{X}$-rays. The value of $\mathrm{T}$ has been compared with the value of BMD: in a previous study we found that the correlation coefficients are 0.80 for the one tenth distal site and 0.83 for a site that is 1 $\mathrm{cm}$ more distal than the previous site. ${ }^{14}$

From the parameters $\mathrm{C}$ and $\mathrm{T}$, measured in millimeters of aluminum, the adimensional ra-

\begin{tabular}{cccc}
$\begin{array}{c}\text { Table 2. Measurements of Thickness and Bone Mineral } \\
\text { Density Values Obtained From Aluminum Steps of } \\
\text { Known Thickness }\end{array}$ \\
$\begin{array}{cccc}\text { Aluminum } \\
(\mathrm{mm})\end{array}$ & $\begin{array}{c}\mathrm{T} \\
\left(\mathrm{mg} / \mathrm{cm}^{2}\right)\end{array}$ & $\begin{array}{c}(\mathrm{Al} \mathrm{mm}) \\
\left(\mathrm{mg} / \mathrm{cm}^{2} \cdot \mathrm{mm} \mathrm{Al}\right)\end{array}$ \\
\hline 1 & 143 & - & 143 \\
1.5 & 200 & 1.54 & 133 \\
2 & 280 & 1.95 & 140 \\
2.5 & 357 & 2.45 & 143 \\
3 & 424 & 2.93 & 141 \\
3.5 & 500 & 3.38 & 143 \\
4 & 574 & 3.85 & 144 \\
4.5 & 626 & 4.33 & 139 \\
5 & 700 & 4.75 & 140 \\
\hline
\end{tabular}

Abbreviations: $T$, thickness; BMD, bone mineral density; $k$, 
tio $\mathrm{C} / \mathrm{T}$ can be derived. Because $\mathrm{C}$ depends mainly on the trabecular texture and $T$ depends on the overall bone tissue, the $\mathrm{C} / \mathrm{T}$ ratio gives an indication of the percentage of the trabecular component with respect to the total bone tissue.

A sample group of 25 women subjected to DPA at one tenth distal of the radius was examined using the procedure described above. Two types of measurements were performed:

\section{Local Measurements}

Three measuring sites were identified on the image of each patient (Fig 3): distal (D), one tenth distal (corresponding to the traditional site of the DPA); very distal (VD), $1 \mathrm{~cm}$ distal with respect to $\mathrm{D}$; and very-very distal (VVD), the proximal line of the distal radioulnar joint. ie, where it is still possible to distinguish the radius from the ulna. For each site, the value of $\mathrm{C}$ along the corresponding scanning line has been calculated according to equation 1 .

\section{Axial Measurements}

Ten subjects whose radiograms showed only slight signs of osteoporosis were excluded from the sample group of 25 patients; the remaining 15 subjects, all postmenopausal women, were divided into two groups: group $\mathrm{A}$, the control group, composed of five patients with no radiological signs of osteoporosis and normal BMD; and group $\mathrm{B}$, composed of 10 patients with evident radiological signs of osteoporosis. The BMD value was within the first SD in four cases. within the second SD in five cases and less than this in just one case.

On the image of each patient in both groups $A$ and $B$, eight rectangular ROIs were identified; each of these ROI is composed of 24 scanning rows, ROI 1 is located at the end of the subchondral hyperdensity band of the articular radius surface (Fig 5 ).

For each $\mathrm{ROI}$, labeled by the index $\mathrm{k}=1,2$, $\ldots 8$ the average value $C_{k}$ has been calculated; on the 12th line of each ROI, $T_{k}$ has been calculated according to equation 3 : for each kth ROI we obtained the ratio $(C / T)_{k}=C_{k} / T_{k}$.

\section{RESULTS AND DISCUSSION}

The results obtained from the local measurements on the sample group of 25 patients are

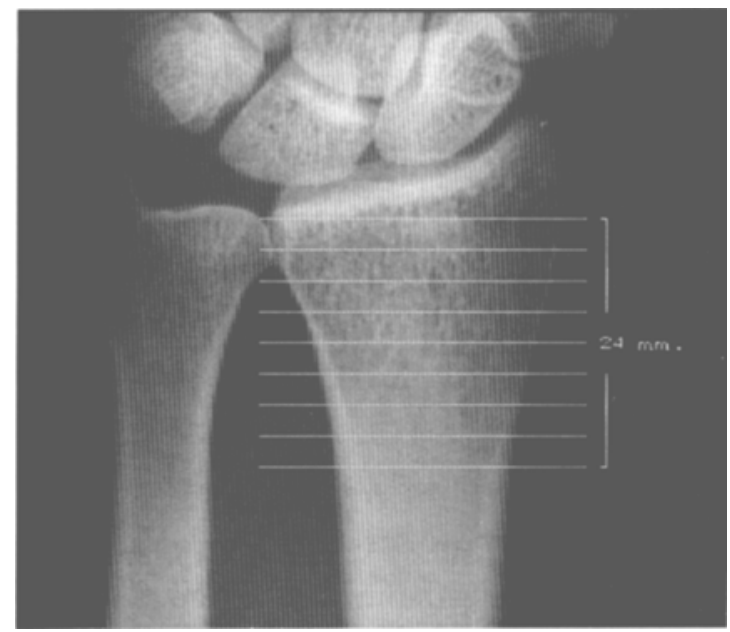

Fig 5. ROls selection for axial measurements: an overall length of $24 \mathrm{~mm}$ is examined.

reported in Tables 3 and 4 . Table 3 contains the measurements of $\mathrm{C}$ for the three sites, Table 4 lists the average values and the relative SDs of the values from Table 3 .

The values are expressed in $10^{-3} \mathrm{~mm} \mathrm{Al}$. The accuracy of the measurements obtained de-

Table 3. Coarseness Measurements on 25 Patients in Distal, Very Distal, and Very-Very Distal Sites

\begin{tabular}{|c|c|c|c|c|c|}
\hline Patient ID & Age & Group & $c_{0}$ & $\mathrm{C}_{\mathrm{vo}}$ & $\mathrm{C}_{\mathrm{vvo}}$ \\
\hline$C B$ & 65 & & 74.6 & 96.4 & 144.0 \\
\hline $\mathrm{CC}$ & 48 & A & 59.3 & 103.3 & 117.3 \\
\hline $\mathrm{Cl}$ & 63 & $B$ & 66.5 & 131.4 & 107.3 \\
\hline DCA & 58 & $B$ & 62.9 & 98.9 & 127.8 \\
\hline DCR & 31 & & 77.2 & 78.1 & 161.8 \\
\hline DLF & 43 & & 78.4 & 106.6 & 137.9 \\
\hline DN & 52 & & 76.5 & 126.1 & 149.4 \\
\hline $\mathrm{FL}$ & 68 & B & 46.0 & 88.0 & 115.8 \\
\hline GN & 61 & & 75.0 & 110.1 & 104.6 \\
\hline LR & 60 & A & 55.8 & 67.5 & 118.9 \\
\hline ME & 18 & & 52.8 & 98.8 & 121.6 \\
\hline MI & 58 & $\mathrm{~B}$ & 64.1 & 114.9 & 112.8 \\
\hline $\mathrm{MM}$ & 52 & & 44.8 & 110.8 & 115.6 \\
\hline NF & 65 & $A$ & 75.7 & 78.3 & 106.3 \\
\hline$P C$ & 57 & & 80.5 & 159.4 & 160.3 \\
\hline PI & 80 & B & 69.5 & 91.0 & 135.7 \\
\hline PW & 75 & B & 57.8 & 109.8 & 123.0 \\
\hline $\mathrm{SC}$ & 81 & B & 141.0 & 159.5 & 141.9 \\
\hline SD & 60 & $B$ & 57.3 & 68.4 & 105.4 \\
\hline SN & 65 & $B$ & 51.3 & 98.2 & 107.1 \\
\hline TA & 62 & & 95.8 & 124.3 & 117.6 \\
\hline $\mathrm{TBi}$ & 64 & A & 70.7 & 94.5 & 107.2 \\
\hline $\mathrm{TBr}$ & 72 & $A$ & 85.1 & 131.9 & 123.3 \\
\hline$T L$ & 67 & & 66.0 & 97.4 & 118.9 \\
\hline VV & 71 & $B$ & 69.4 & 104.7 & 113.3 \\
\hline
\end{tabular}

Abbreviations: ID, identification; $C$, coarseness; D, distal; VD, very distal; VVD, very-very distal. 
Table 4. Mean Values and Standard Deviations for Parameter C

\begin{tabular}{cccrccr}
\hline Item & $\overline{\mathrm{C}}_{\mathrm{D}}$ & $\mathrm{SD}$ & \multicolumn{1}{c}{$\overline{\mathrm{C}}_{\mathrm{vD}}$} & \multicolumn{1}{c}{$\mathrm{SD}$} & $\overline{\mathrm{C}}_{\mathrm{vvD}}$ & \multicolumn{1}{c}{$\mathrm{SD}$} \\
\hline Sample & 70.2 & 19.2 & 105.9 & 23.6 & 125.0 & 18.7 \\
Group A & 69.3 & 12.0 & 95.1 & 24.8 & 114.6 & 7.5 \\
Group B & 68.6 & 26.8 & 106.5 & 25.1 & 119.0 & 12.7 \\
\hline
\end{tabular}

Abbreviations $C$, coarseness; $D$, distal; SD, standard deviation; VD, very distal; VVD, very-very distal.

pends both on the precision of the xy scanner used to derive the photometric values and also on the position of the forearm during the x-ray examination. In fact, if the forearm is rotated on its own longitudinal axis, different projections of the same bone can be radiographed. Thus, when the $x$-ray is analyzed, the data input to the system can vary, and different results can be obtained. On the other hand, positions of the forearm that involve an inclination of the longitudinal axis on the $\mathrm{x}$-ray field are compensated by registering the $\mathrm{x}$-ray before digitizing it so that the measuring sites can remain as lines perpendicular to the longitudinal axis of the bone. However, in this way it is still possible to have variations in the measures because of the subjective localization of the reference line on the subchondral surface made by the user. These two types of errors, which depend on the torsion of the forearm or on the user interaction, are evaluated either by repeating the analysis of the same $x$-ray with a different operator or by analyzing $\mathrm{x}$-rays of the same patient taken at different times. This type of statistical investigation has still not been performed and, consequently, we can only report the measuring error introduced by the xy scanner: the measuring precision is $\pm 0.5 \times 10^{-3}$ Almm and it is obtained by analyzing the data reported in Table 1 .

Table 3 shows that the value of $\mathrm{C}$ increases from the distal site to the VVD site in all 25 cases. Assuming that $\mathrm{C}$ depends on the trabecular content of the radiographed bone structure, the measurements obtained using our method are in agreement with those found by Nilas, ${ }^{15}$ who calculated the relative content of the trabecular bone from 13 necropsies.

Applying the test of significance to the difference between the mean values for groups $A$ and $\mathrm{B}$, in Table 4 we obtain the values $t=0.052$ in site $\mathrm{D}, t=0.951$ in the VD site and $t=0.665$ in the VVD site. These values are considerably below Student's $t$ index for 13 degrees of freedom, which is equivalent to $t=2.160$ for a level of significance of $95 \%$. For this reason we cannot yet affirm that the difference between the means is significant, despite the fact that the results obtained indicate that the most suitable site for the analyses proposed is the VD site.

A diminution of the number and an increase in the density of the remaining trabeculae can be noted in the radiograms of osteoporotic subjects. The value given to $\mathrm{C}$, which depends mainly on the trabecular component, makes it possible to quantify the information gathered from the radiograms, even though the results of the method followed to obtain $C$ show a certain degree of indetermination.

In fact, to derive $\mathrm{C}$, a filter is applied to the acquired signal and the power spectrum of the filtered signal is measured. Because there is, in general, a spatial frequency interval in which the power spectrum depends exclusively on the trabecular pattern, the filtering operation can introduce an error that cannot be estimated with precision without making a further comparative study with istomorphometric measurements.

The postmenopausal osteoporotic process is characterized both by a diminution in the total bone mineral content and by a variation in the percentage of the trabecular content. The value of $\mathrm{C}$ by itself is thus not sufficient to quantify the mineral loss. The $\mathrm{C} / \mathrm{T}$ ratio is far more suitable for this purpose because it also takes the total bone content into account.

The value of $T$ depends both on the cortical and on the trabecular structure. However, $T$ is also dependent on the nonbone components. The contribution given by these to the attenuation of the $\mathrm{x}$-ray imaging has in fact only been eliminated to a certain extent, using a function obtained by linear interpolation between two points on the graph of a scanning line of the image. Consequently, an estimate of the percentage of the trabecular component from the $\mathrm{C} / \mathrm{T}$ ratio is affected by a measuring inaccuracy. Nevertheless, although it is not equivalent to the istomorphometric measurement of the percentage of trabecular bone content, $\mathrm{C} / \mathrm{T}$ supplies a useful estimate for diagnostic purposes.

The graph in Fig 6 shows the mean values of 
$\mathrm{C} / \mathrm{T}$ and the SDs derived from the set of values for groups $\mathrm{A}$ and $\mathrm{B}$, respectively.

The values of $C$ / $T$ for group $B$ are found to be consistently higher than those for group $\mathrm{A}$. C/T thus provides a correct estimate of the percentage of trabecular bone content which is higher in osteoporotic subjects (group B) than in the control group. In both groups, $\mathrm{C} / \mathrm{T}$ increase starting from the subchondral region, reach their maximum in ROI2, at approximately 4 to 6 $\mathrm{mm}$ from the surface of the subchondral bone of the radius, and then decrease in proximal direction. This trend is similar to that of the curve derived by Schlenker ${ }^{16}$ from a postmortem examination of four women of different ages, by separating and weighing the trabecular and cortical bones extracted from sections of the radius and the ulna.

In ROI8 (approximately one tenth the radius), the mean $\mathrm{C} / \mathrm{T}$ values for the two groups are closer; the curves diverge in a more evident manner in correspondence with ROI3 and $\mathrm{ROI} 4$, ie, at 7 to $12 \mathrm{~mm}$ from the first measuring row.

The curve for group A shows a slight slope from ROI8 to ROI3 and a sharp increase from ROI3 to ROI2. This is in agreement with the aspect of the X-ray image of healthy subjects, in which the trabecular bone is significantly represented in correspondence with the area con-

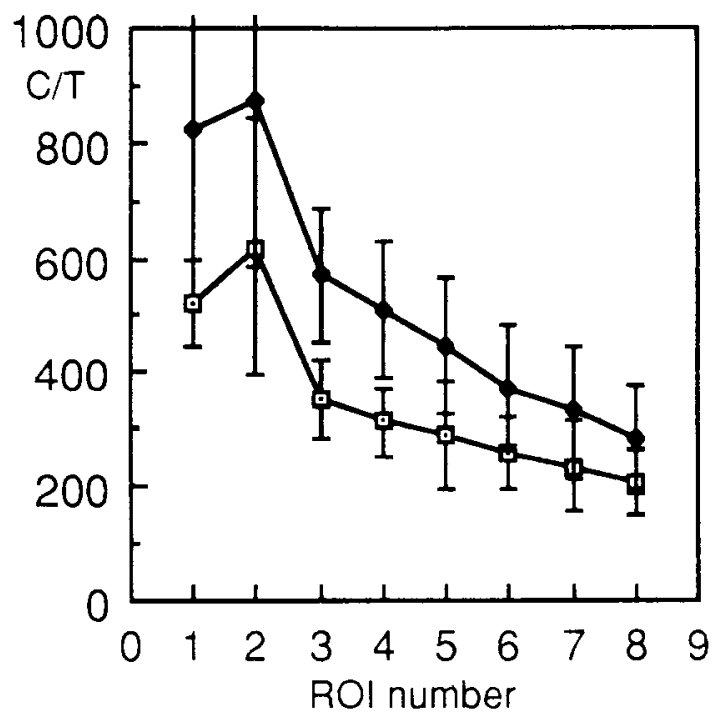

Fig 6. Graph of the axial measurements: an overall length of $24 \mathrm{~mm}$ is examined, subdivided into eight adjacent ROls. tained between ROI2 and ROI3. On the contrary, the curve for group B slopes more sharply, in agreement with the increase in the trabecular bone which can be seen on the x-ray image of osteoporotic subjects, starting from a more proximal site with respect to the healthy subjects.

\section{CONCLUSIONS}

The identification of the measuring ROIs depends on the operator, who must first select a reference line on the digital image; the ROIs are then determined automatically on the rows of the image, at a preestablished distance from it. Because of the high spatial resolution in the digital image acquired, the variations that can occur when an expert operator identifies the anatomic detail to be taken as the reference point are contained within a pair of pixels. The effective position of the ROIs can be translated by just a single pair of pixels. Because each ROI consists of 24 adjacent lines, the $\mathrm{C} / \mathrm{T}$ values obtained by translating the reference line of \pm 2 pixels can be ignored.

Despite the errors introduced during the interactive stage, the method proposed for the numerical analysis of conventional x-ray images has been shown to be adequate for the study of the pattern determined by the bone structure on radiographs of the distal portion of the radius. Furthermore, although our sample was limited, the results obtained are encouraging as far as the application of this method to the study of bone pathology is concerned. This method makes it possible to evaluate the reduction in the trabeculae and the increase in their thickness.

Parameter $\mathrm{C}$ can be used as an index for quantifying the pattern of the trabecular bone. The measurements performed have shown that $\mathrm{C}$ is in agreement with the results of anatomic and istomorphometric studies on the structure of the trabecular bone of the distal portion of the radius.

Parameter $\mathrm{C} / \mathrm{T}$ has been found to be a valid index for the evaluation of the percentage of the bone trabecular content. In particular, $\mathrm{C} / \mathrm{T}$ allowed us to discriminate between pathological and healthy subjects. In our study, the most significant variations in the percentage of the 
trabecular bone content expressed by $\mathrm{C} / \mathrm{T}$ were found to be located approximately 6 to $12 \mathrm{~mm}$ from the first measuring row: in this region, the values are separated by more than two SDs.

If the method proposed is to be used for clinical evaluations (eg, in the screening of asymptomatic patients or in the diagnosis and grading of symptomatic patients), the dimensions of the errors associated with changes in position of the forearm must be estimated.

\section{REFERENCES}

1. Cann EC: Skeletal structure-function revisited. Radiology 179:607-608, 1991

2. Griffiths HJ, Virtama P: Cortical thickness and trabecular pattern of the femoral neck as a measure of osteopenia. Invest Radiol 25:1116-1119, 1990

3. Phillips HB, Owen JS, Chandler B: Quantitative histology of bone: A computerized method for measuring the total mineral content of bone. Calcif Tiss Res 26:85-89, 1978

4. Colbert C, Mazess RB, Schmidt PB: Bone mineral determination in vitro by radiographic photodensitometry and direct photon absorptiometry. Invest Radiol 5:336-340, 1970

5. Rockoff SD, Scandrett J, Zacher R: Quantization of relevant image information: Automated radiographic bone trabecular characterization. Radiology 101:435-439, 1971

6. Durand EP, Ruegsegger P: Cancellous bone structure: Analysis of high-resolution CT images with the run-length method. J Comp Assist Tomogr 15:133-139, 1991

7. Trouerbach WT, Grashuis JL, Zwamborn AW, et al: Microdensitometric analysis of bone structures in $x$-ray images. Skeletal Radiol 16:190-195, 1987

8. Hesp R, Deacon AC, Hulme $P$, et al: Trends in trabecular and cortical bone in the radius compared with whole body calcium balance in osteoporosis. Clin Sci 66:109. 112,1984

9. Nilas L, Borg J, Gotfredsen A, et al: Comparison of single and dual photon absorptiometry in postmenopausal bone mineral loss. J Nucl Med 26:1257-1262, 1985

10. Kalebo P, Strid KG: Bone mass determination from microradiographs by computer-assisted videodensitometry: II-Aluminium as a reference substance. Acta Radiol 29:611617,1988

11. Bevington PR: Data Reduction and Error Analysis for the Physical Sciences. New York, NY, McGraw-Hill, 1969

12. Lang P, Steiger P, Faulkner K, et al: Osteoporosis. Current techniques and recent developments in quantitative bone densitometry. Radiol Clin North Am 29:49-76, 1991

13. Johns HE, Cunningham JR: The Physics of Radiology. Springfield, IL, Thomas, 1983

14. Chimenti M, Trippi D, Bozzi R, et al: Computer characterization of trabecular bone patterns, in Lemke HU, et al (eds): Proc CAR '89. New York, NY, Springer, 1989

15. Nilas L, Nørgaard H, Pødenphant J, et al: Bone composition in the distal forearm. Scan J Clin Lab Invest $47: 41-46,1987$

16. Schlenker RA: Percentages of cortical and trabecular bone mineral mass in the radius and ulna. in Mazess RB (ed): Third Int Conf Bone Mineral Measurement. AJR Am J Roentgenol 126:1309-1312, 1976 